\title{
Comparison of postoperative pain and pain control techniques in uniportal and biportal VATS and open surgery patients
}

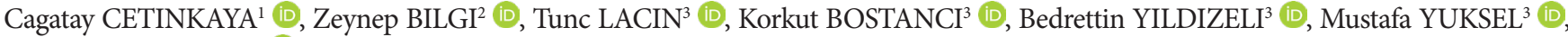 \\ Hasan Fevzi BATIREL ${ }^{3}$ \\ ${ }^{1}$ Thoracic Surgery Clinic, Memorial Atasehir Hospital, Atasehir, Istanbul, Turkey. \\ ${ }^{2}$ Department of Thoracic Surgery, School of Medicine, Medeniyet University, Kadikoy, Istanbul, Turkey. \\ ${ }^{3}$ Department of Thoracic Surgery, School of Medicine, Marmara University, Pendik, Istanbul, Turkey.
}

\author{
Corresponding Author: Cagatay CETINKAYA \\ E-mail: drcagataycet@gmail.com
}

Submitted: 03.01.2021 Accepted: 21.04.2021

\begin{abstract}
Objectives: Thoracotomy causes intense postoperative pain which may become chronic. Video-assisted thoracic surgery (VATS) leads to less postoperative pain compared with thoracotomy. In this study, we analyzed pain scores in patients who underwent lung resections with VATS or thoracotomy.

Patients and Methods: Patients who underwent lung resections with uniportal, biportal VATS or thoracotomy between May 2015

- May 2017 were included in the study. Visual Analogue Scale (VAS) pain scores were recorded on postoperative day 1, 5 (or at discharge), 2nd week, 1st and 3rd months. Patients were classified in 3 groups, uniportal VATS $(n=178)$, biportal VATS ( $\mathrm{n}=15)$, thoracotomy $(\mathrm{n}=60)$. Demographics, resection type, mortality, morbidity and epidural catheter use were recorded.

Results: Two hundred and fifty-three patients (average age was $57.3 \pm 12.7,94$ females) were included in the study. Median hospital stay was 5 days. Uniportal and biportal groups had significantly lower pain scores in all intervals compared with thoracotomy. No chronic pain was seen in VATS groups. Uniportal and biportal groups had similar pain scores at all times. Epidural use or size of specimen did not affect pain in VATS patients ( $\mathrm{p}=0.18 \mathrm{vs} \mathrm{p}=0.68)$.

Conclusion: Video-assisted thoracic surgery decreases the need for epidural patient control analgesia. Specimen size does not affect postoperative pain and chronic pain is rare.

Keywords: Thoracoscopy, Uniportal, Biportal, Postoperative analgesia
\end{abstract}

\section{INTRODUCTION}

Pain control after thoracic operations is of critical importance. Thoracotomy causes intense postoperative pain, which may require epidural analgesia and/or opioid medications (morphine, oxycontine, tramadol etc.) [1]. Postoperative intensive pain control approaches may hinder the patient's compliance to pulmonary rehabilitation, inadequate ambulation may lead to atelectasis and pneumonia. Pain may still persist after discharge, affecting patient's toleration for adjuvant treatments, cause poor quality of life and also chronic pain syndromes may occur in $5-10 \%$ of patients who underwent thoracotomy [2].
Video-assisted thoracic surgery (VATS) has become the standard of care in the last 15 years for early stage non-small cell lung cancer (NSCLC). The reason of this popularity is mainly due to decreased postoperative pain with VATS compared to thoracotomy. This has been reported in comparative and randomized studies which mainly use visual analogue scale (VAS) for pain quantification [3,4]. Improvements in VAS scores have also been correlated with functional gains after thoracic surgery [5].

Video-assisted thoracic surgery approach can be used for various procedures ranging from anatomical resections to pleural

How to cite this article: Cetinkaya C, Bilgi Z, Lacin T, et al. Comparison of postoperative pain and pain control techniques in uniportal - biportal VATS and open surgery patients. Marmara Med J 2021; 34(3):260-266. doi: 10.5472/marumj.984215 
biopsies. For anatomical resections, standard VATS approach uses 3 ports, but as the technique gained popularity and surgeons completed their learning curve, biportal and uniportal anatomic resections have been reported in large case series. Comparative studies for those procedures are being published and the functional gain of these patients (if any) is not clear $[6,7]$. There is also no currently established guideline delineating the use of basic (medication) or advanced (epidural, paravertebral) pain control modalities in various VATS techniques.

The aim of this study is to compare three different thoracic surgical techniques (uniportal VATS, biportal VATS, thoracotomy) and the size of lung specimens (wedge resection vs anatomical resection) in terms of pain scores, necessity of epidural analgesia and presence of chronic pain.

\section{PATIENTS and METHODS}

\section{Patient selection}

All patients undergoing anatomical or limited lung resection between May 2015-May 2017 were prospectively enrolled in this study. Patients were evaluated in 3 groups; Uniportal VATS $(n=178)$, biportal VATS $(n=15)$ and thoracotomy (open surgery) $(n=60)$. All patients received standard preoperative work up according to their condition (lung cancer, solitary pulmonary nodule, bronchiectasis etc.) and anesthetic needs (routine laboratory studies, chest X-ray, electrocardiogram, pulmonary function tests, required consults according to present comorbidities). Patients with pre-diagnosed chronic pain syndromes or on chronic opioids and patients who did not consent to participate or could not cooperate for the application of the VAS were excluded. Demographic data, medical history (comorbidities, previous surgery, malignancy, etc), operative data (type of surgery, presence of epidural catheter, etc), perioperative data (length of hospital stay, opioid use during hospital and after discharge) and VAS for pain were prospectively recorded in a database.

The study was approved by the Marmara University, School of Medicine Clinical Research Ethics Committee on 02.12.2016 with the protocol code of 09.2016.589. Per regulations of the review board and the hospital, written consent was taken from each patient.

\section{Surgical technique}

Uniportal VATS incision was made on the anterior axillary line, at the 5th intercostal space. $3-5 \mathrm{~cm}$ incision was made and a wound protecting retractor was used in every patient. A 28-32 $\mathrm{F}$ drain was placed through the same incision at the end of the operation.

Biportal VATS approach included a second incision in addition to the incision described in uniportal technique, which was usually at the 7 th or 8 th intercostal space, on the anterior axillary line. A 28-32 F drain was placed through the lower port at the end of the operation.
Open surgery was performed through a classical posterolateral or anterolateral thoracotomy through 5 th intercostal space. Rib retractor was used in all cases. 2 chest drains were placed from separate incisions.

\section{Analgesic technique and follow up}

All patients enrolled in the study received either intravenous (pethidine) or epidural (bupivacaine) patient controlled analgesia (PCA). Pethidine PCA settings were loading dose of $0.6 \mathrm{mg} / \mathrm{kg}$, and a request dose of $0.2 \mathrm{mg} / \mathrm{kg}$ with basal infusion of $0.1 \mathrm{mg} / \mathrm{kh} /$ hour. An analgesic solution of bupivacaine $(0.125 \%)$ was used for epidural PCA. In both groups, locked out time was set as 30 minutes. Thoracic epidural catheters were placed preoperatively in the operating theater, just before the operation. Anesthesiology postoperative pain team followed up the PCAs for 3 days every 12 hours and per needed basis in between. After postoperative day 3, analgesic maintenance was through oral and IV medications (tramadol, acetaminophen, ibuprofen). PCA was terminated on the day of discharge if the hospital stay was shorter than 3 days.

Patients were provided with a single script for 20 tramadol tablets, $50 \mathrm{mg}$, taken up to thrice daily as needed at discharge. They were instructed to rely on diclofenac $75 \mathrm{mg}$ (twice a day) and acetaminophen $500 \mathrm{mg}$ tablets (thrice a day) first, before using tramadol.

\section{Visual analogue scale (VAS) pain scores}

Visual Analogue Scale is an established method used for pain quantification. In our study the type of VAS that was provided had a 1 to 10 scale with 1 as pain free and 10 as the worst pain imaginable. The scale also included graphical references, pictured as representative faces at that corresponding pain level [8]. The measurements were taken on postoperative 1st, 5th days and on 2nd week, 1st month and 3rd month.

\section{Statistical Analysis}

Data were analyzed using SPSS 22.0. $\mathrm{p}<0.05$ was determined as statistically significant. Mean, standard deviation and median values were calculated accordingly.

Distribution of variables were determined by using Kolmogorov - Smirnov test. Qualitative independent variables were evaluated by using Kruskal-Wallis and Mann-Whitney-U tests. Wilcoxon test was used for dependent qualitative parameters. For quantitative assessment, Chi-Square was used when conditions were met, otherwise Fisher's exact test was performed.

\section{RESULTS}

\section{Patient characteristics}

Among the patients included in the study, 178 patients underwent uniportal, 15 patients biportal VATS and the remaining 60 patients underwent thoracotomy. Operation breakdown is shown in Table I. 
Mean age was $57.3 \pm 12.7$. There was no statistically significant age difference between groups (Table I).

Epidural cathater PCA was applied to 183 patients (114 uniportal, 12 biportal, 57 open), while in 70 patients analgesia was provided (64 uniportal, 3 biportal, 3 open) via IV PCA (Table I).

Uniportal and biportal groups had significantly lower pain scores on all intervals when compared to open group, however on the postoperative day 14 , although the difference was evident, it was not statistically significant between biportal and open surgery groups (p:0.087). The difference in pain scores between these 3 groups was most pronounced in postoperative day (POD) 1 $(3.3 \pm 1.6,3.3 \pm 1.3$ vs $4.7 \pm 1.1 \mathrm{p}<0.001)$. (Table II, Figure1).

On postoperative day 5 , significantly lower percentage of patients in uniportal group scored higher than 4 when compared to open group (18/160 [11.3\%] vs 17/60 [28.3\%], p=0.01).

In chronic phase (3rd month) 14 uniportal, 3 biportal and 27 open surgery patients had very low pain intensity. While none of the uniportal and biportal patients described significant pain at 3rd month, 4 open surgery patients still had medium pain intensity. $(0.1 \pm 0.4,0.3 \pm 0.6$ vs $0.8 \pm 1.1 \mathrm{p}<0.001)$.

The subgroup analysis in regards to operation type (anatomic resection vs non anatomic resection) did not yield to any statistically significant different results in terms of VAS score composition (Table III). Also, in uniportal cases epidural and IV PCA groups were comparable in all intervals regardless of the operation type (Table IV).

\section{Length of hospital stay}

Uniportal surgery group had statistically a shorter length of hospital stay when compared to open surgery group (4.7 \pm 3.3 , $5.5 \pm 2.2$ vs $7.3 \pm 4.1 \mathrm{p}<0.001)$. There was no significant difference in regards to the length of hospital stay in either uniportal versus biportal or biportal versus open surgery comparisons.

\section{Opioid usage after discharge}

While a greater percentage of open surgery patients filled in and used their opioid (tramadol $50 \mathrm{mg}, 20$ tablets, thrice a day) prescsriptions than uniportal patients $(27 \%$ vs $11 \%, \mathrm{p}<0.04)$, biportal group's opioid consumption was comparable to uniportal and open surgery groups $(\mathrm{p}>0.05)$.

Table I. Patient characteristics, operation and analgesic type of subgroups

\begin{tabular}{|l|c|c|c|}
\hline & $\begin{array}{c}\text { Uniportal } \\
(\mathrm{n}=178)\end{array}$ & $\begin{array}{c}\text { Biportal } \\
(\mathrm{n}=15)\end{array}$ & $\begin{array}{c}\text { Open surgery } \\
(\mathrm{n}=60)\end{array}$ \\
\hline $\begin{array}{l}\text { Age (mean } \pm \text { SD, } \\
\text { Median) }\end{array}$ & $\begin{array}{c}56.8 \pm 12.4, \\
58.0\end{array}$ & $63.7 \pm 9.1,63.0$ & $57.0 \pm 13.9,59.5$ \\
\hline $\begin{array}{l}\text { Sex }(\mathrm{n}, \%) \text { Female } \\
\mathrm{N}=72,40 \% \\
\mathrm{~N}=106,59 \%\end{array}$ & $\begin{array}{c}\mathrm{N}=3,20 \% \\
\mathrm{~N}=12,80 \%\end{array}$ & $\begin{array}{c}\mathrm{N}=19,31 \% \\
\mathrm{~N}=41,68 \%\end{array}$ \\
\hline $\begin{array}{l}\text { Anatomic resection } \\
(\mathrm{n}, \%)\end{array}$ & $\mathrm{N}=89,50 \%$ & $\mathrm{~N}=14,93.3 \%$ & $\mathrm{~N}=50,90 \%$ \\
\hline $\begin{array}{l}\text { Wedge resection } \\
(\mathrm{n}, \%)\end{array}$ & $\mathrm{N}=89,50 \%$ & $\mathrm{~N}=1,6.7 \%$ & $\mathrm{~N}=6,10 \%$ \\
\hline $\begin{array}{l}\text { Analgesic control } \\
\text { Epidural PCA } \\
(\mathrm{n}, \%) \quad \text { IV PCA }\end{array}$ & $\mathrm{N}=114,64 \%$ & $\mathrm{~N}=12,80 \%$ & $\mathrm{~N}=57,95 \%$ \\
\hline
\end{tabular}

PCA: Patient Controlled Analgesia

Table II. Distribution of VAS pain scores according to the groups at different intervals

\begin{tabular}{|c|c|c|c|c|c|c|c|c|c|c|c|c|c|c|}
\hline & \multicolumn{5}{|c|}{ Uniportal } & \multicolumn{3}{|c|}{ Biportal } & \multicolumn{4}{|c|}{ Open Surgery } & \multirow{2}{*}{\multicolumn{2}{|c|}{$\mathrm{p}$}} \\
\hline & & ean \pm s.d. & & Median & & Mean \pm s.d. & & Median & & Mean \pm s.d. & & Median & & \\
\hline \multicolumn{15}{|l|}{ VAS } \\
\hline Postop Day 1 & 3.3 & \pm & 1.6 & 3.0 & 3.3 & \pm & 1.3 & 4.0 & 4.7 & \pm & 1.1 & 4.5 & $<0.001$ & $\mathrm{~K}$ \\
\hline Postop Day 5 & 1.9 & \pm & 1.1 & 2.0 & 2.2 & \pm & 1.0 & 2.0 & 3.0 & \pm & 0.9 & 3.0 & $<0.001$ & ${ }^{\mathrm{K}}$ \\
\hline Postop $2^{\text {nd }}$ week & 0.6 & \pm & 0.8 & 0.0 & 1.1 & \pm & 0.9 & 1.0 & 1.6 & \pm & 0.7 & 2.0 & $<0.001$ & K \\
\hline Postop $1^{\text {st }}$ month & 0.2 & \pm & 0.6 & 0.0 & 0.2 & \pm & 0.4 & 0.0 & 0.9 & \pm & 0.8 & 1.0 & $<0.001$ & $\mathrm{~K}$ \\
\hline Postop $3^{\text {rd }}$ month & 0.1 & \pm & 0.4 & 0.0 & 0.3 & \pm & 0.6 & 0.0 & 0.8 & \pm & 1.1 & 0.0 & $<0.001$ & $\mathrm{~K}$ \\
\hline
\end{tabular}

K Kruskal-Wallis, VAS: Visual Analogue Scale

Table III. Distribution of VAS pain scores in terms of resection types

\begin{tabular}{|c|c|c|c|c|c|c|c|c|c|c|}
\hline & & & Wed & & & & tomic & ion & & \\
\hline & & Mean. \pm s.d. & & Median & & Mean \pm s.d. & & Median & & \\
\hline VAS & & & & & & & & & & \\
\hline Postop Day 1 & 3.27 & \pm & 1.69 & 3.00 & 3.37 & \pm & 1.42 & 4.00 & 0.172 & $\mathrm{~m}$ \\
\hline Postop Day 5 & 1.88 & \pm & 1.05 & 2.00 & 1.94 & \pm & 1.16 & 2.00 & 0.555 & $\mathrm{~m}$ \\
\hline Postop $2^{\text {nd }}$ week & 0.46 & \pm & 0.66 & 0.00 & 0.71 & \pm & 0.89 & 0.00 & 0.091 & $\mathrm{~m}$ \\
\hline Postop $1^{\text {st }}$ month & 0.17 & \pm & 0.48 & 0.00 & 0.30 & \pm & 0.65 & 0.00 & 0.143 & $\mathrm{~m}$ \\
\hline Postop $3^{\text {rd }}$ monthW & 0.03 & \pm & 0.18 & 0.00 & 0.17 & \pm & 0.48 & 0.00 & 0.052 & $\mathrm{~m}$ \\
\hline
\end{tabular}

m Mann-Whitney U test, VAS: Visual Analogue Scale 
Table IV. Distribution of VAS pain scores between epidural and IV PCA in uniportal incision

\begin{tabular}{|c|c|c|c|c|c|c|c|c|c|c|}
\hline & \multicolumn{5}{|c|}{ Epidural (-) } & \multicolumn{3}{|c|}{ Epidural $(+)$} & \multirow{2}{*}{\multicolumn{2}{|c|}{$\mathrm{P}$}} \\
\hline & & $1 \pm$ s.d. & & Median & & Mean \pm s.d. & & Median & & \\
\hline \multicolumn{11}{|l|}{$V A S$} \\
\hline Postop Day 1 & 3.17 & \pm & 1.45 & 3.00 & 3.40 & \pm & 1.61 & 3.50 & 0.335 & $\mathrm{~m}$ \\
\hline Postop Day 5 & 1.70 & \pm & 0.90 & 2.00 & 2.03 & \pm & 1.19 & 2.00 & 0.092 & $\mathrm{~m}$ \\
\hline Postop $2^{\text {nd }}$ week & 0.45 & \pm & 0.75 & 0.00 & 0.66 & \pm & 0.81 & 0.00 & 0.078 & $\mathrm{~m}$ \\
\hline Postop $1^{\text {st }}$ month & 0.08 & \pm & 0.27 & 0.00 & 0.32 & \pm & 0.67 & 0.00 & 0.009 & $\mathrm{~m}$ \\
\hline Postop $3^{\text {rd }}$ month & 0.06 & \pm & 0.30 & 0.00 & 0.12 & \pm & 0.40 & 0.00 & 0.242 & $\mathrm{~m}$ \\
\hline
\end{tabular}

m Mann-Whitney U test

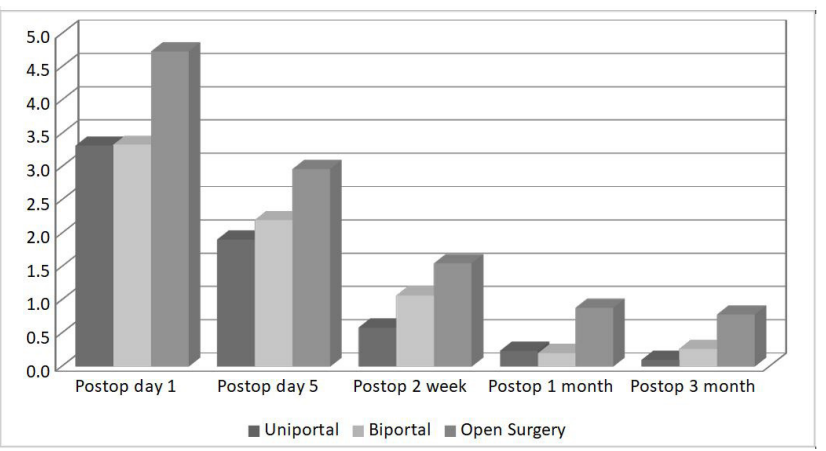

Figure 1. VAS pain scores of three groups at different intervals

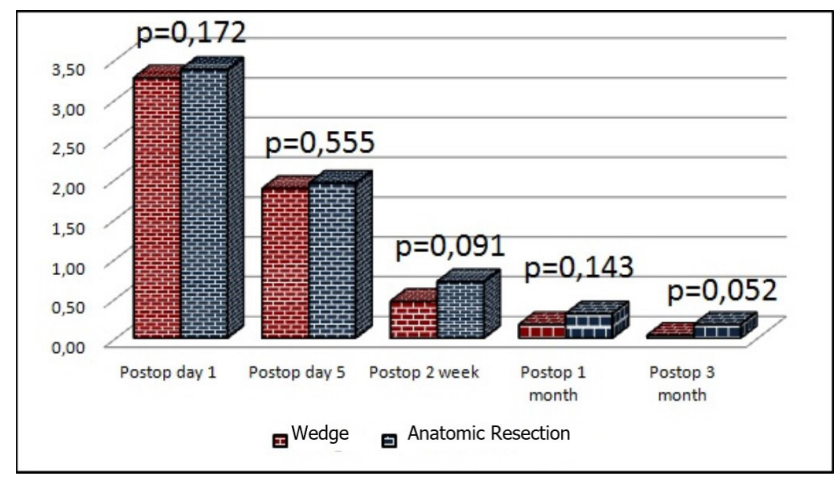

Figure 2. Distribution of VAS pain scores according to the resection types

\section{DISCUSSION}

A total of 253 patients who have undergone uniportal and biportal VATS and thoracotomy for various indications were included in this study. No significant difference was found in terms of pain between the uniportal and the biportal groups at all times except from the resection size comparison. Although, there was no significant difference between biportal and open surgery at the postoperative 2nd week, uniportal and biportal groups had lower pain scores compared with the open surgical group at all other times (Figure 1).

The first comparison of postoperative pain in VATS versus thoracotomy approaches was done by Landreneau et al., in 1993, which concluded in favor of VATS [9]. After that, various articles elaborated on postoperative pain and other aspects like length of hospital stay, length of chest drains, complications, quality of life, survival etc. 39 of those studies were reviewed in a meta-analysis by Whitson et al., among 6370 patients. VATS was found to be superior in terms of postoperative complications, length of chest drainage and hospital stay [3]. Since, the nature of the problem makes it very difficult to design a double blind randomized study and recruit adequate number of patients, the National Comprehensive Cancer Network guidelines now accept VATS as a first line approach for early state NSCLC, citing meta-analysis and well designed propensity score match studies show both superiority and non-inferiority to thoracotomy in regards to perioperative and oncological outcomes [10].

Enhanced recovery after surgery (ERAS) pathways were initially developed in colorectal surgery. In a meta-analysis of 38 studies, ERAS pathways were seen to be effective in reducing hospital length of stay and postoperative complication rates [11]. Guidelines for enhanced ERAS have recently been published for lung surgery. A standardized multimodal approach to pain relief, including good regional anaesthesia, is recommended with the aim of reducing postoperative opioid use. Acetaminophen and non-steroidal anti-inflammatory drugs should be administered regularly to all patients unless contraindications exist [12]. The use of ERAS after lung surgery has the potential to improve patient outcomes. Early mobilization and VATS lung resection were independently associated with reduced morbidity while early mobilization was the only independent factor influencing a reduced length of hospital stay [13]. Our standard approach was to have patient walk in-room after $6^{\text {th }}$ postoperative hour, right after introduction of a liquid diet. Since, the time of the disposition of the patient (family home, skilled nursing facility, rehabilitation facility, assisted living, etc.) is dependent on the practice environment we were not able to directly compare our 
hospital stay statistics but 5-7 days is within the range of the established literature $[7,12,13]$.

Video-assisted surgery is a versatile approach, allowing application to a wide range of pathologies with different modifications. Uniportal VATS became popular after 2008 and some articles compared uniportal vs multiportal approaches in terms of postoperative pain and opioid consumption. While Jutley et al., found uniportal VATS to be more favourable in pneumothorax cases in acute postoperative period, uniportal and multiportal approaches were found to be equivalent in the chronic phase [14]. McElnay et al., found both approaches comparable when the population of the study was limited to anatomic resections [15]. This report's cohort, regarding the range of pathologies covered with VATS and postoperative pain outcomes, was comparable in both acute and chronic phases regardless of the number of the incisions.

In another study, similar to ours, by Sebastian et al., patients were divided into 3 groups: robotic surgery, VATS and open surgery and pain scores were compared. There was not a significant difference between VATS and robotic-assisted thoracoscopic surgery (RATS) groups in terms of acute and chronic pain and pain scores were less than the open surgery group in the acute period. However, different from our study, in the chronic period, there was not a significant difference in pain scores between 3 groups [16].

Although, the pathogenesis of pain is not clear, the most common opinion is that postthoracotomy pain is caused by intercostal nerve injury. Pain can sometimes exist after 4 to 5 years in $30 \%$ of patients [17]. In our study, during the chronic pain evaluation in the third month: no patients in the uniportal $(0 / 178)$ and biportal $(0 / 15)$ groups had pain, while in the open surgery group (4/60) 4 patients had moderate pain (Table II, Figure 1). The reason of this pain is thought to be nerve injury caused by intercostal retractions during surgery.

Epidural analgesia, paravertebral blocks, intercostal nerve blocks, PCA are the most common used pain control methods after thoracic surgery. Jie Ae Kim et al., compared the epidural PCA and paravertebral block methods for pain control in 37 patients who underwent VATS during the postoperative 5 days period and there was no significant difference between pain levels and pulmonary functions [18]. Epidural PCA or IV PCA were applied to all patients in our study. Pain scores were evaluated in 2 groups named as epidural PCA and IV PCA in 178 patients who had uniportal resection. There was not a significant difference in pain levels between these groups in our study (Table IV). Our study revealed that lung resections with uniportal incisions result in lower pain scores and pain control was comparable with systemic or epidural pain control methods in accordance with the reported experiences of different clinics in the literature $[19,20]$. We compared the patients in 2 groups according to the size of resection: named as wedge resection and anatomical resection. The results showed that the size of resection has no effect on pain. There are no studies in literature comparing the size of the specimen and its relevance with postoperative pain.
Thoracic surgery is associated with high level of pain and an elevated incidence of long term opioid use after surgery [21]. Opioid use is also associated with significant risk for both mobidity and mortality [22].There are no clinical studies which analyze opioid needs after discharge following lung cancer surgery at different types of incision. However, many news and studies derived from especially the USA $[23,24]$ show that the increase in the number of IV opioid users and the change in their demographic profile in these days are caused by the opioid prescriptions during discharge and their easy access. Due to this reason, it is better for patient safety to choose the methods that do not require opioid consumption in discharge. Our study showed that in the postoperative fifth day ( which is usually the day of discharge) patients who had 4 and more in pain scores in uniportal VATS group were significantly low. Since, sociocultural factors affect pain resistance and analgesic requirements, in our study opioid consumption was overall low in all groups. However, if this is not the case in other cultures, uniportal VATS may be a solution to decrease opioid consumption.

This study has several limitations. First of all, this study is not randomized and possibility of selection bias cannot be excluded. Thoracotomy patients may have had more advanced stage tumors. Secondly, the number of patients are not evenly distributed. Biportal patients are usually conversions from uniportal VATS due to difficulty in stapler introduction or lung manipulation. The study also does not have a power analysis, thus some of the significances may have been unnoticed due to the limited number of patients. Additionally, our study is terminated with 3 month follow-up and we could not assess pain scores at 6 months and 1 year postoperatively.

In conclusion, this study shows that VATS technique, independent of the number of port incisions, may decrease or eliminate the need for an epidural PCA for postoperative analgesia, specimen size does not affect postoperative pain and chronic pain is not highly expected in VATS patients.

\section{Compliance with Ethical Standards}

Ethical approval: Ethical approval was obtained from the Marmara University Faculty of Medicine Clinical Research Ethics Committee on 02.12.2016 with the protocol code of 09.2016.589. Per regulations of the review committee and the hospital, written consent was taken from each patient.

Funding: The study was not supported by any funds.

Conflict of Interest: The authors have no conflicts of interest to declare.

Author Contributions: C.C. Substantial contributions to the conception or design of the work; or the acquisition, analysis, or interpretation of data for the work, Agreement to be accountable for all aspects of the work in ensuring that questions related to the accuracy or integrity of any part of the work are appropriately investigated and resolved. Final approval of the version to be published. Z.B. Drafting the work or revising it critically for important intellectual content, Substantial contributions to the conception or design of the work; or the acquisition, analysis, or interpretation of data for the work, Final approval of the version 
to be published. T.L. Drafting the work or revising it critically for important intellectual content, Final approval of the version to be published. K.B. Drafting the work or revising it critically for important intellectual content, Final approval of the version to be published. B.Y. Drafting the work or revising it critically for important intellectual content, Final approval of the version to be published. M.Y. Drafting the work or revising it critically for important intellectual content, Final approval of the version to be published. H.F.B. Drafting the work or revising it critically for important intellectual content, Final approval of the version to be published. Agreement to be accountable for all aspects of the work in ensuring that questions related to the accuracy or integrity of any part of the work are appropriately investigated and resolved.

\section{REFERENCES}

[1] Ochroch EA, Gottschalk A, Augostides J, et al. Long-term pain and activity during recovery from major thoracotomy using thoracic epidural analgesia. Anesthesiology $2002 ; 97: 1234-44$. doi: 10.1097/00000.542.200211000-00029.

[2] Karmakar MK, Ho AM. Postthoracotomy pain syndrome. Thorac Surg Clin 2004 ;14:345-52. doi: 10.1016/S15474127(04)00022-2

[3] Whitson BA, Groth SS, Duval SJ, Swanson SJ, Maddaus MA. Surgery for early-stage non-small cell lung cancer: a systematic review of the video-assisted thoracoscopic surgery versus thoracotomy approaches to lobectomy. Ann Thorac Surg. 2008 ;86:2008-16; discussion 2016-8. doi: 10.1016/j. athoracsur.2008.07.009

[4] Bendixen M, Jørgensen OD, Kronborg C, Andersen C, Licht PB. Postoperative pain and quality of life after lobectomy via video-assisted thoracoscopic surgery or anterolateral thoracotomy for early stage lung cancer: a randomised controlled trial. Lancet Oncol 2016 ;17:836-844. doi: 10.1016/ S1470-2045(16)00173-X

[5] Varela G, Brunelli A, Rocco G, et al. Predicted versus observed FEV1 in the immediate postoperative period after pulmonary lobectomy. Eur J Cardiothorac Surg 2006 ;30:6448. doi: 10.1016/j.ejcts.2006.07.001

[6] Rizk NP, Ghanie AM, Meier H, et al. A prospective trial comparing pain and quality of life measures after anatomic lung resection using either thoracoscopy or thoracotomy. Ann Thorac Surg 2014 ; 98: 1160-66. doi: 10.1016/j. athoracsur.2014.05.028

[7] Nomori H, Cong Y, Sugimura H. Limited thoracotomy for segmentectomy: a comparison of postoperative pain with thoracoscopic lobectomy. Surg Today 2016 ;46:1243-8. doi: 10.1007/s00595.015.1302-4

[8] Langley GB, Sheppeard H. The visual analogue scale: its use in pain measurement. Rheumatol Int 1985;5:145-8. doi: 10.1007/ BF00541514.

[9] Landreneau RJ, Mack MJ, Hazelrigg SR, et al. Prevalence of chronic pain after pulmonary resection by thoracotomy or video-assisted thoracic surgery. J Thorac Cardiovasc Surg
1994;107:1079-86. doi: 10.1097/00132.586.19941200000051

[10] Sihoe ADL. "Video-assisted thoracoscopic surgery as the gold standard for lung cancer surgery." Respirology 2020; 25: 49-60.

[11] Nicholson A, Lowe MC, Parker J, Lewis SR, Alderson P, Smith AF. Systematic review and meta-analysis of enhanced recovery programmes in surgical patients. Br J Surg 2014; 101:172-88. doi: 10.1002/bjs.9394

[12] Batchelor TJP, Rasburn NJ, Abdelnour-Berchtold E, et al. Guidelines for enhanced recovery after lung surgery: recommendations of the Enhanced Recovery After Surgery (ERAS1) Society and the European Society of Thoracic Surgeons (ESTS). Eur J Cardiothorac Surg 2019;55:91-115. doi: 10.1093/ejcts/ezy301

[13] Batchelor TJP, Ljungqvist O. A surgical perspective of ERAS guidelines in thoracic surgery. Curr Opin Anaesthesiol 2019;32:17-22. doi: 10.1097/ACO.000.000.0000000685

[14] Jutley RS, Khalil MW, Rocco G. Uniportal vs standard threeport VATS technique for spontaneous pneumothorax: comparison of postoperative pain and residual paraesthesia. Eur J Cardiothorac Surg 2005;28: 43-6. doi: 10.1016/j. ejcts.2005.02.039

[15] McElnay PJ, Molyneux M, Krishnadas R, Batchelor TJP, West $\mathrm{D}$, Casali G. Pain and recovery are comparable after either uniportal or multiport videoassisted thoracoscopic lobectomy: an observation study. Eur J Cardiothorac Surg 2015 ;47:912-5. doi: 10.1093/ejcts/ezu324

[16] Kwon ST, Zhao L, Reddy RM, et al. Evaluation of acute and chronic pain outcomes after robotic, video-assisted thoracoscopic surgery, or open anatomic pulmonary resection. J Thorac Cardiovasc Surg 2017 ;154:652-9. doi: 10.1016/j. jtcvs.2017.02.008

[17] Karmakar MK, Ho AM. Postthoracotomy pain syndrome. Thorac Surg Clin 2004 ;14:345-52. doi: 10.1016/S15474127(04)00022-2

[18] Kim JA, Kim TH, Yang M, et al. Is intravenous patient controlled analgesia enough for pain control in patients who underwent thoracoscopy? J Korean Med Sci 2009 ;24:930-5. doi: 10.3346/jkms.2009.24.5.930

[19] Haager B, Schmid D, Eschbach J, Passlick B, Loop T. Regional versus systemic analgesia in video-assisted thoracoscopic lobectomy: a retrospective analysis. BMC Anesthesiology 2019; 19: 1-9.

[20] Yie J C, Yang JT, Wu C Y, Sun W Z, Cheng Y J. Patient-controlled analgesia (PCA) following video-assisted thoracoscopic lobectomy: comparison of epidural PCA and intravenous PCA. Acta Anaesthesiologica Taiwanica 2012; 50:92-95.

[21] Kavanagh BP, Katz J, Sandler AN. Pain control after thoracic surgery. A review of current techniques.Anesthesiology 1994 ;81:737-59. doi: 10.1097/00000.542.199409000-00028

[22] Huang A, Azam A, Segal S, et al. Chronic postsurgical pain and persistent opioid use following surgery: the need for a transitional pain service. Pain Manag 2016 ;6:435-43. doi: 10.2217/pmt-2016-0004 
[23] Bates C, Laciak R, Southwick A, Bishoff J. Overprescription of postoperative narcotics: a look at postoperative pain medication delivery, consumption and disposal in urological practice. J Urol 2011;185:551-5 doi: 10.1016/j.juro.2010.09.088
[24] Alam A, Gomes T, Zheng H , Mamdani MM, Juurlink DN, Bell CM. Long-term analgesic use after low-risk surgery: a retrospective cohort study. Arch Intern Med. Arch Intern Med 2012;172:425-30. doi: 10.1001/archinternmed.2011.1827 